\title{
Comment avoir des conversations difficiles avec vos patients
}

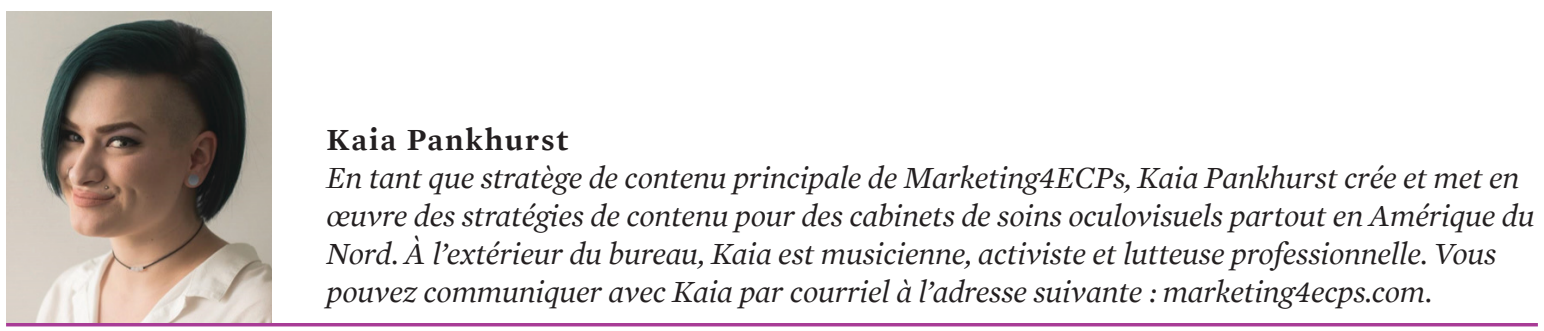

En tant que stratège de contenu principale de Marketing4ECPs, Kaia Pankhurst crée et met en œuvre des stratégies de contenu pour des cabinets de soins oculovisuels partout en Amérique du pouvez communiquer avec Kaia par courriel à l'adresse suivante : marketing4ecps.com.

$\mathrm{C}$ omme tout professionnel de la santé, les optométristes doivent parfois aborder des sujets difficiles avec leurs patients. Qu'il s'agisse d'un diagnostic effrayant, d'une garantie de monture expirée ou même de mauvaises nouvelles au sujet de la couverture d'assurance, ces conversations sont un élément indissociable de leur travail.

Ni le patient ni le praticien ne se réjouit d'avoir ce genre de discussions, mais les occulter ne constitue pas une option. La meilleure chose à faire est d'aborder les sujets difficiles avec compassion, élégance et clarté.

Voici quelques stratégies que vous pouvez employer lorsque vous avez des conversations difficiles avec vos patients.

\section{ÉTABLIR LES GRANDES LIGNES DE LA CONVERSATION}

Préparez-vous à la discussion avant de l'entamer. Commencez par déterminer l'essence de votre message. Que devezvous communiquer? Couvrez l'information nécessaire, idéalement en quelques phrases courtes, puis ouvrez un dialogue.

Une des méthodes que vous pourriez envisager est la stratégie «question-réponse-question », où vous demandez au patient quelle est sa principale préoccupation, répondez à sa question, puis faites un suivi en lui demandant s'il estime que vous avez répondu à la question.

Pour un plan plus complet, essayez d'utiliser la stratégie SPIKES :

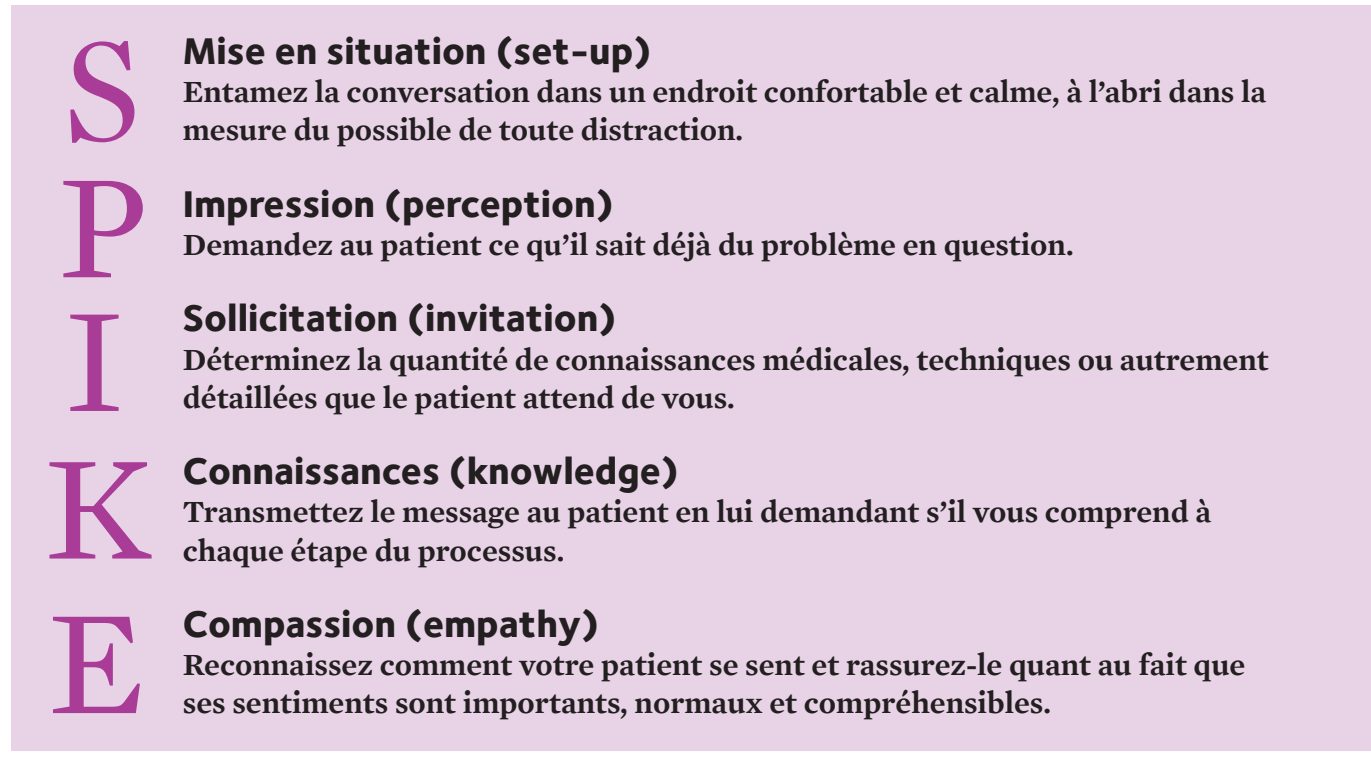


Demandez à votre patient s'il a des questions. Une fois que vous avez répondu à chaque question, confirmez auprès de ce dernier que vos réponses à ses questions lui conviennent. Une fois que le patient se sent prêt, vous pouvez lui dire quelles seront les prochaines étapes de son traitement.

\section{ORIENTER LE PATIENT VERS DES RESSOURCES}

De toute évidence, vous ne pouvez pas offrir un soutien émotionnel complet à tous vos patients. Mais vous pouvez les aider à trouver un système de soutien à l'extérieur de votre bureau. Faites quelques recherches et créez une base de données de groupes de soutien, de mentors, d'organismes de bienfaisance et d'autres organisations qui offrent de l'aide et du soutien à des personnes comme votre patient.

En montrant à votre patient qu'il n'est pas seul, vous pouvez l'aider à faire la transition vers la nouvelle réalité qui est maintenant la sienne. Vous lui montrez ainsi que vous vous souciez de sa santé et de son bien-être en général.

\section{RESTER PRÉSENT}

L’empathie et la vulnérabilité émotionnelle peuvent être des sentiments parfois désagréables à éprouver. De nombreux professionnels de la santé ont tendance à couper court à leurs impulsions plus « humaines » au cours de conversations difficiles, en utilisant du jargon médical ou technique au lieu de s'engager dans un dialogue.

Bien qu'il puisse s'agir d'une réaction naturelle, elle peut troubler, effrayer ou frustrer davantage les patients.

Il est important de rester présent sur le plan émotionnel dans le cadre de tels scénarios. Communiquez l'information dans des termes simples. Expliquez les termes médicaux et essayez d'établir des analogies, le cas échéant, pour ajouter du contexte. Faites tout ce que vous pouvez pour interagir avec votre patient plutôt que de lui présenter des informations. Soyez ouvert à l'idée de dire « Je suis désolé », lorsque c'est approprié.

\section{NE PAS OUBLIER QUE VOUS ÊTES AUSSI UN ÊTRE HUMAIN}

Les conversations difficiles ont des répercussions sur toutes les personnes concernées. Si vous voulez communiquer des mauvaises nouvelles à un patient, vous avez aussi besoin de soutien émotionnel. Qu'il s'agisse d'amis, de membres de votre famille ou de collègues, assurez-vous de disposer d'un réseau de personnes qui peuvent vous offrir de l'aide et vous donner de la force dans les moments difficiles.

Prenez le temps d'analyser les sentiments qui vous habitent. Participez à des activités qui vous redonnent de l'énergie et vous permettent d'offrir à vos patients la meilleure expérience possible. 